\title{
Fuzzy Multi-sphere Support Vector Data Description
}

\author{
Trung Le, Dat Tran, Wanli Ma, and Dharmendra Sharma \\ Faculty of Information Sciences and Engineering \\ University of Canberra, ACT 2601, Australia
}

\begin{abstract}
Multi-sphere Support Vector Data Description (MSSVDD) has been proposed in our previous work. MS-SVDD aims to build a set of spherically shaped boundaries that provide a better data description to the normal dataset and an iterative learning algorithm that determines the set of spherically shaped boundaries. MS-SVDD could improve classification rate for oneclass classification problems comparing with SVDD. However MS-SVDD requires a small abnormal data set to build the spherically shaped boundaries for the normal data set. In this paper, we propose a new fuzzy MS-SVDD that can be used when only the normal data set is available. Experimental results on 14 well-known datasets and a comparison between fuzzy MS-SVDD and SVDD are also presented.
\end{abstract}

Index Terms-Novelty detection, one-class classification, support vector data description, and fuzzy model.

\section{INTRODUCTION}

Support Vector Data Description (SVDD) is a new Support Vector Machine (SVM) learning method for one-class classification [1]. A hyperspherically shaped boundary around the normal dataset is constructed to separate this set from abnormal data. The volume of this data description is minimized to reduce the chance of accepting abnormal data. SVDD has been proven as one of the best methods for one-class classification problems [2].

Some extensions to SVDD have been proposed to improve the margins of the hyperspherically shaped boundary. The first extension is Small Sphere and Large Margin (SSLM) [3] which proposes to surround the normal data in this optimal hypersphere such that the margin-distance from outliers to the hypersphere, is maximized. This SSLM approach is helpful for parameter selection and provides very good detection results on a number of real datasets. We have recently proposed a further extension to SSLM which is called Small Sphere and Two Large Margins (SS2LM) [4]. This SS2LM aims at maximising the margin between the surface of the hypersphere and abnormal data and the margin between that surface and the normal data while the volume of this data description is being minimised.

Other extensions to SVDD regarding data distribution have also been proposed. The first extension is to apply SVDD to multi-class classification problems [5]. Several class-specific hyperspheres that each encloses all data samples from one class but excludes all data samples from other classes. The second extension is for one-class classification which proposes to use a number of hyperspheres to decribe the normal dataset [2]. Normal data samples may have some distinctive distributions so they will locate in different regions in the feature space and hence if the single hypersphere in SVDD is used to enclose all normal data, it will also enclose abnormal data samples resulting a high false positive error rate. However this work was not presented in detail, the proposed method is heuristic and there is no proof provided to show that the multisphere approach can provide a better data description.

In our previous work [6], we proposed Multi-sphere SVDD (MS-SVDD) as a new multi-hypersphere approach to SVDD. A set of hyperspheres was proposed to describe the normal dataset assuming that normal data samples have distinctive data distributions. We formulated the optimisation problem for multi-sphere SVDD and proved how SVDD parameters were obtained through solving this problem. An iterative algorithm was also proposed for building data descriptors, and we also proved that the classification error will be reduced after each iteration.

However MS-SVDD requires a small abnormal data set to build the spherically shaped boundaries for the normal data set. To overcome this problem, we propose in this paper a new fuzzy MS-SVDD that can be used when only the normal data set is available. Experimental results on 14 well-known datasets in UCI repository show that the proposed method provides lower classification error rates comparing with the standard single-sphere SVDD.

\section{Support Vector Data Description (SVDD)}

Let $X=\left\{x_{1}, x_{2}, \ldots, x_{n}\right\}$ be the dataset. SVDD [1] aims at determining an optimal sphere including all normal data points in this dataset $X$ while abnormal data points are not included. The optimisation problem is as follows

$$
\min _{R, c, \xi}\left(R^{2}+\frac{1}{\nu n} \sum_{i=1}^{n} \xi_{i}\right)
$$

subject to

$$
\begin{aligned}
& \left\|\phi\left(x_{i}\right)-c\right\|^{2} \leq R^{2}+\xi_{i}, \quad i=1, \ldots, n \\
& \xi_{i} \geq 0, \quad i=1, \ldots, n
\end{aligned}
$$

where $R$ is radius of the hypersphere, $c$ is centre of the sphere, $\xi=\left[\xi_{i}\right]_{i=1, \ldots, n}$ is vector of slack variables, $\nu$ is a positive constant, $\phi($.$) is the nonlinear function related to the symmetric,$ positive definite kernel function $K\left(x_{1}, x_{2}\right)=\phi\left(x_{1}\right)^{T} \phi\left(x_{2}\right)$.

For classifying an unknown data point $x$, the following decision function is used: $f(x)=\operatorname{sign}\left(R^{2}-\|\phi(x)-c\|^{2}\right)$. The unknown data point $x$ is normal if $f(x)=+1$ or abnormal if $f(x)=-1$. 


\section{FuZzy Multi-SPhere Support Vector Data DESCRIPTION (FMS-SVDD)}

\section{A. Problem Formulation}

Consider a set of $m$ hyperspheres $S_{j}\left(c_{j}, R_{j}\right)$ with center $c_{j}$ and radius $R_{j}, j=1, \ldots, m$. This hypersphere set is a good data description of the normal dataset $X=\left\{x_{1}, x_{2}, \ldots, x_{n}\right\}$ if each of the hyperspheres describes a distribution in this dataset and the sum of all radii $\sum_{j=1}^{m} R_{j}^{2}$ should be minimised.

Let matrix $U=\left[u_{i j}\right]_{n \times m}, u_{i j} \in[0,1], i=1, \ldots, n, j=$ $1, \ldots, m$ where $u_{i j}$ is the membership representing degree of belonging of data point $x_{i}$ to hypersphere $S_{j}$. The optimisation problem of fuzzy multi-sphere SVDD (FMS-SVDD) can be formulated as follows

$$
\min _{R, c, \xi}\left(\sum_{j=1}^{m} R_{j}^{2}+\frac{1}{\nu n} \sum_{i=1}^{n} \xi_{i}\right)
$$

subject to

$$
\sum_{\substack{j=1 \\ \xi_{i} \geq 0, \quad i=1, \ldots, n}}^{m} u_{i j}^{\alpha}\left\|\phi\left(x_{i}\right)-c_{j}\right\|^{2} \leq \sum_{j=1}^{m} u_{i j}^{\alpha} R_{j}^{2}+\xi_{i}, \quad i=1, \ldots, n
$$

where $R=\left[R_{j}\right]_{j=1, \ldots, m}$ is vector of radii, $\nu$ is constant, $\xi_{i}$ are slack variables, and $c=\left[c_{j}\right]_{j=1, \ldots, m}$ is vector of centres.

In FMS-SVDD model, we also introduce parameter $\alpha \geq$ 1 to adjust the relative ratios among fuzzy memberships $u_{i j}, j=1, \ldots, m$.

Minimising the function in (3) over variables $R, c$ and $\xi$ subject to (4) will determine radii and centres of hyperspheres and slack variables if the matrix $U$ is given. On the other hand, the matrix $U$ will be determined if radii and centres of hyperspheres are given. Therefore an iterative algorithm will be applied to find the complete solution. The algorithm consists of two alternative steps: 1) Calculate radii and centres of hyperspheres and slack variables, and 2) Calculate membership $U$.

We present in the next sections the iterative algorithm and prove that the classification error in the current iteration will be smaller than that in the previous iteration.

For classifying a data point $x$, the following decision function is used

$$
f(x)=\operatorname{sign}\left(\max _{1 \leq j \leq m}\left\{R_{j}^{2}-\left\|\phi(x)-c_{j}\right\|^{2}\right\}\right)
$$

The unknown data point $x$ is normal if $f(x)=+1$ or abnormal if $f(x)=-1$. This decision function implies that the mapping of a normal data point has to be in one of the hyperspheres and that the mapping of an abnormal data point has to be outside all of those hyperspheres. The following theorem is used to consider the relation of slack variables to data points classified.

Theorem 1: Assume that $(R, c, \xi)$ is a solution of the optimisation problem in (3), $x_{i}, i \in\{1,2, \ldots, n\}$ is the $\mathrm{i}$-th data point. The slack variable $\xi_{i}$ can be computed as

$$
\xi_{i}=\max \left\{0, \sum_{j=1}^{m} u_{i j}^{\alpha}\left(\left\|\phi\left(x_{i}\right)-c_{j}\right\|^{2}-R_{j}^{2}\right)\right\}
$$

Proof: For all $i$, from (4) we have

$$
\xi_{i} \geq \max \left\{0, \sum_{j=1}^{m} u_{i j}^{\alpha}\left(\left\|\phi\left(x_{i}\right)-c_{j}\right\|^{2}-R_{j}^{2}\right)\right\}
$$

Moreover, $(R, c, \xi)$ is minimal solution of (3). Hence, $\xi_{i}=$ $\max \left\{0, \sum_{j=1}^{m} u_{i j}^{\alpha}\left(\left\|\phi\left(x_{i}\right)-c_{j}\right\|^{2}-R_{j}^{2}\right)\right\}$.

The following empirical error can be defined for a data point $x_{i}$

$\operatorname{error}(i)=\left\{\begin{array}{l}0 \text { if } \mathrm{x}_{i} \text { is correctly classified } \\ \sum_{j=1}^{m} u_{i j}^{\alpha}\left(\left\|\phi\left(x_{i}\right)-c_{j}\right\|^{2}-R_{j}^{2}\right) \text { if otherwise }\end{array}\right.$

Referring to Theorem 1, it is easy to prove that $\sum_{i=1}^{n} \xi_{i}$ is an upper bound of $\sum_{i=1}^{n} \operatorname{error}(i)$.

\section{B. Calculating Radii, Centres and Slack Variables}

The Lagrange function for the optimisation problem in in (3) subject to (4) is as follows

$$
\begin{aligned}
& L(R, c, \xi, \alpha, \beta)=\sum_{j=1}^{m} R_{j}^{2}+\frac{1}{\nu n} \sum_{i=1}^{n} \xi_{i}+ \\
& \sum_{i=1}^{n} \alpha_{i}\left(\sum_{j=1}^{m} u_{i j}^{\alpha}\left(\left\|\phi\left(x_{i}\right)-c_{j}\right\|^{2}-R_{j}^{2}\right)-\xi_{i}\right) \\
& -\sum_{i=1}^{n} \beta_{i} \xi_{i}
\end{aligned}
$$

Setting derivatives of $L(R, c, \xi, \alpha, \beta)$ with respect to primal variables to 0 , we obtain

$$
\frac{\partial L}{\partial R_{j}}=0 \Rightarrow \sum_{i=1}^{n} \alpha_{i} u_{i j}^{\alpha}=1, \quad j=1, \ldots, m
$$

$$
\begin{aligned}
& \frac{\partial L}{\partial c_{j}}=0 \Rightarrow \sum_{i=1}^{n} \alpha_{i} u_{i j}^{\alpha}\left(\phi\left(x_{i}\right)-c_{j}\right)=0 \\
& \Rightarrow c_{j}=\sum_{i=1}^{n} \alpha_{i} u_{i j}^{\alpha} \phi\left(x_{i}\right), \quad j=1, \ldots, m
\end{aligned}
$$

$$
\frac{\partial L}{\partial \xi_{j}}=0 \Rightarrow \alpha_{i}+\beta_{i}=\frac{1}{\nu n}, \quad i=1, \ldots, n
$$


To get the dual form, we substitute (10)-(12) to the Lagrange function in (9) and obtain the following

$$
\begin{aligned}
& L=\sum_{i=1}^{n} \sum_{j=1}^{m} \alpha_{i} u_{i j}^{\alpha}\left\|\phi\left(x_{i}\right)-c_{j}\right\|^{2} \\
& =\sum_{i=1}^{n} \sum_{j=1}^{m} \alpha_{i} u_{i j}^{\alpha} K\left(x_{i}, x_{i}\right)-2 \sum_{i=1}^{n} \sum_{j=1}^{m} \alpha_{i} u_{i j}^{\alpha} \phi\left(x_{i}\right) c_{j} \\
& +\sum_{i=1}^{n} \sum_{j=1}^{m} \alpha_{i} u_{i j}^{\alpha}\left\|c_{j}\right\|^{2} \\
& =\sum_{i=1}^{n} \sum_{j=1}^{m} \alpha_{i} u_{i j}^{\alpha} K\left(x_{i}, x_{i}\right)-2 \sum_{j=1}^{m} c_{j} \sum_{i=1}^{n} \alpha_{i} u_{i j}^{\alpha} \phi\left(x_{i}\right) \\
& +\sum_{j=1}^{m}\left\|c_{j}\right\|^{2} \sum_{i=1}^{n} \alpha_{i} u_{i j}^{\alpha} \\
& =\sum_{i=1}^{n} \sum_{j=1}^{m} \alpha_{i} u_{i j}^{\alpha} K\left(x_{i}, x_{i}\right)-2 \sum_{j=1}^{m}\left\|c_{j}\right\|^{2}+\sum_{j=1}^{m}\left\|c_{j}\right\|^{2} \\
& =\sum_{i=1}^{n} \alpha_{i} K\left(x_{i}, x_{i}\right) \sum_{j=1}^{m} u_{i j}^{\alpha}-\sum_{j=1}^{m}\left\|c_{j}\right\|^{2} \\
& =\sum_{i=1}^{n} K\left(x_{i}, x_{i}\right) \mathrm{v}_{i} \alpha_{i}-\sum_{j=1}^{m}\left(\sum_{i=1}^{n} u_{i j}^{\alpha} \alpha_{i} \phi\left(x_{i}\right)\right)^{2} \\
& =\sum_{i=1}^{n} K\left(x_{i}, x_{i}\right) \mathrm{v}_{i} \alpha_{i}-\sum_{j=1}^{m} \sum_{1 \leq i, k \leq n} u_{k j}^{\alpha} u_{i j}^{\alpha} K\left(x_{i}, x_{k}\right) \alpha_{i} \alpha_{k} \\
& =\sum_{i=1}^{n} K\left(x_{i}, x_{i}\right) \mathrm{v}_{i} \alpha_{i}-\sum_{1 \leq i, k \leq n} K\left(x_{i}, x_{k}\right) \alpha_{i} \alpha_{k} \sum_{j=1}^{m} u_{k j}^{\alpha} u_{i j}^{\alpha} \\
& =\sum_{i=1}^{n} K\left(x_{i}, x_{i}\right) \mathrm{v}_{i} \alpha_{i}-\sum_{1 \leq i, k \leq n} K\left(x_{i}, x_{k}\right) r_{i k} \alpha_{i} \alpha_{k}
\end{aligned}
$$

where $r_{i k}=\sum_{j=1}^{m} u_{k j}^{\alpha} u_{i j}^{\alpha}, \quad 1 \leq i, k \leq n$ and $v_{i}=$ $\sum_{j=1}^{m} u_{i j}^{\alpha}, \quad 1 \leq i \leq n$.

It leads to the following quadratic optimisation problem

$$
\min _{\alpha}\left(\sum_{1 \leq i, k \leq n} K\left(x_{i}, x_{k}\right) r_{i k} \alpha_{i} \alpha_{k}-\sum_{i=1}^{n} K\left(x_{i}, x_{i}\right) \mathrm{v}_{i} \alpha_{i}\right)
$$

subject to

$$
\begin{aligned}
& \sum_{i=1}^{n} u_{i j}^{\alpha} \alpha_{i}=1, \quad j=1, \ldots, m \\
& 0 \leq \alpha_{i} \leq \frac{1}{\nu n}, \quad i=1, \ldots, n
\end{aligned}
$$

\section{Calculating Membership $U$}

We are in position to describe how to evaluate matrix $\mathrm{U}$ after obtaining new $(R, c)$. Given $1 \leq i \leq n$, let us denote

$$
\begin{aligned}
& d_{i j}=\left\|\phi\left(x_{i}\right)-c_{j}\right\|^{2}-R_{j}^{2} \quad \text { and } \quad D_{i j}=\left(\frac{1}{d_{i j}}\right)^{\frac{1}{\alpha-1}} \\
& j_{0}=\underset{1 \leq j \leq m}{\arg \min } d_{i j}
\end{aligned}
$$

The membership matrix can be updated as follows

If $d_{i j_{0}} \leq 0$ then $u_{i j_{0}}=1$, and $u_{i j}=0\left(j \neq j_{0}\right)$ Else

$$
u_{i j}=\frac{D_{i j}}{\sum_{k=1}^{n} D_{k j}}, \quad i, j=1, \ldots, n
$$

\section{Iterative Learning Process}

The proposed iterative learning process for multi-sphere SVDD will run two alternative steps until a convergence is reached as follows

\section{Initialise $U$ by clustering the normal dataset in the input space \\ Repeat the following \\ Calculate $R, c$ and $\xi$ using $U$ \\ Calculate $U$ using $R$ and $c$ \\ Until a convergence is reached}

\section{E. Theoretical Background of FMS-SVDD}

In the objective function $\sum_{j=1}^{m} R_{j}^{2}+\frac{1}{\nu n} \sum_{i=1}^{n} \xi_{i}$, the first sum can be regarded as regularization quantity and the second one can be considered as empirical risk. We will prove that the structural risk $\sum_{j=1}^{m} R_{j}^{2}+\frac{1}{\nu n} \sum_{i=1}^{n} \xi_{i}$ becomes smaller in Theorem 3.

Theorem 2: Given a $n$-multivariate function $f\left(x_{1}, x_{2}, \ldots, x_{n}\right)=\sum_{i=1}^{n} d_{i} x_{i}^{\alpha}$. The following optimisation problem

$$
\min _{x} f(x)
$$

subject to

$$
\sum_{i=1}^{n} x_{i}=1
$$

yields the solution as follows

If $d_{i_{0}} \leq 0$ then $x_{i_{0}}=1$ and $x_{i}=0\left(i \neq i_{0}\right)$

Else $x_{i}=\frac{\left(\frac{1}{d_{i}}\right)^{\frac{1}{\alpha-1}}}{\sum_{k=1}^{n}\left(\frac{1}{d_{k}}\right)^{\frac{1}{\alpha-1}}}, \quad i=1, \ldots, n$
where $i_{0}=\underset{1 \leq i \leq n}{\arg \min } d_{i}$.

Proof:

Case 1: $d_{i_{0}}<0$

$\sum_{i=1}^{n} d_{i} x_{i}^{\alpha} \geq d_{i_{0}} \sum_{i=1}^{n} x_{i}^{\alpha} \geq d_{i_{0}} \sum_{i=1}^{n} x_{i}=d_{i_{0}}=f\left(0, \ldots, 1_{i_{0}}, \ldots, 0\right)$

since $\alpha>1$.

Case 2: $d_{i_{0}} \geq 0$ The Lagrange function is of

$$
L(x, \lambda)=\sum_{i=1}^{n} d_{i} x_{i}^{\alpha}-\lambda\left(\sum_{i=1}^{n} x_{i}-1\right)
$$

where $\lambda$ is Lagrange multiplier.

Setting derivatives to 0 , we gain

$$
\begin{aligned}
& \frac{\partial L}{\partial x_{i}}=0 \Rightarrow \alpha d_{i} x_{i}^{\alpha-1}-\lambda=0 \\
& \Rightarrow x_{i}=\left(\frac{\lambda}{\alpha d_{i}}\right)^{\frac{1}{\alpha-1}}, \quad i=1, \ldots, n
\end{aligned}
$$

From $\sum_{i=1}^{n} x_{i}=1$, we have $x_{i}=\frac{\left(\frac{1}{d_{i}}\right)^{\frac{1}{\alpha-1}}}{\sum_{k=1}^{n}\left(\frac{1}{d_{k}}\right)^{\frac{1}{\alpha-1}}}, \quad i=$ 
The following theorem straight forward reasons the aforementioned statement

Theorem 3: Let $\left(R^{(t)}, c^{(t)}, \xi^{(t)}, U^{(t)}\right)$ and $\left(R^{(t+1)}, c^{(t+1)}\right.$, $\left.\xi^{(t+1)}, U^{(t+1)}\right)$ be solutions at the previous iteration and current iteration, respectively. The following inequality holds

$$
\sum_{j=1}^{m}\left(R_{j}^{(t+1)}\right)^{2}+\frac{1}{\nu n} \sum_{i=1}^{n} \xi_{i}^{(t+1)} \leq \sum_{j=1}^{m}\left(R_{j}^{(t)}\right)^{2}+\frac{1}{\nu n} \sum_{i=1}^{n} \xi_{i}^{(t)}
$$

Proof: By referring to Theorem 2, it is easy to see that $u^{(t+1)}=\left(u_{i 1}^{(t+1)}, \ldots, u_{i m}^{(t+1)}\right)$ is solution of the following optimisation problem

$$
\min \left(\sum_{j=1}^{m} d_{i j}^{(t)} u_{i j}^{\alpha}\right)
$$

subject to

$$
\sum_{j=1}^{m} u_{i j}=1
$$

Therefore, we have

$$
\sum_{j=1}^{m} d_{i j}^{(t)}\left(u_{i j}^{(t+1)}\right)^{\alpha} \leq \sum_{j=1}^{m} d_{i j}^{(t)}\left(u_{i j}^{(t)}\right)^{\alpha}
$$

It means that

$$
\begin{aligned}
& \sum_{j=1}^{m}\left(u_{i j}^{(t+1)}\right)^{\alpha}\left(\left\|\phi\left(x_{i}\right)-c_{j}^{(t)}\right\|^{2}-\left(R_{j}^{(t)}\right)^{2}\right) \\
& \leq \sum_{j=1}^{m}\left(u_{i j}^{(t)}\right)^{\alpha}\left(\left\|\phi\left(x_{i}\right)-c_{j}^{(t)}\right\|^{2}-\left(R_{j}^{(t)}\right)^{2}\right) \leq \xi_{i}^{(t)}
\end{aligned}
$$

or

$\sum_{j=1}^{m}\left(u_{i j}^{(t+1)}\right)^{\alpha}\left\|\phi\left(x_{i}\right)-c_{j}^{(t)}\right\|^{2} \leq \sum_{j=1}^{m}\left(u_{i j}^{(t+1)}\right)^{\alpha}\left(R_{j}^{(t)}\right)^{2}+\xi_{i}^{(t)}$

Hence, $\left(R^{(t)}, c^{(t)}, \xi^{(t)}, U^{(t)}\right)$ is feasible solution of optimisation problem (3) at time $t+1$. Since $\left(R^{(t+1)}, c^{(t+1)}, \xi^{(t+1)}\right.$, $\left.U^{(t+1)}\right)$ is minimal solution of this optimisation problem, theorem 3 is proved.

\section{EXPERIMENTAL RESUlts}

In this section, we present our experiment performed on 14 datasets in UCI repository as shown in Table I. For each dataset, we selected one class and appointed it as normal class. The other class is regarded as the abnormal one. We run cross validation with five folds and compared FMS-SVDD with SVDD. It is noted that we will not compare with our previous non-fuzzy MS-SVMM as this model requires a small set of abnormal data for learning. In the experiment performed for this paper, no abnormal data set is provided in the training process, therefore the classification rate provided by the nonfuzzy one will be lower than that by the proposed fuzzy model FMS-SVDD in this paper.

The popular RBF Kernel $K\left(x, x^{\prime}\right)=e^{-\gamma\left\|x-x^{\prime}\right\|^{2}}$ was applied whereas the parameter $\gamma$ was varied in grid $\left\{2^{i}: i=\right.$ $2 j+1, j=-8, \ldots, 1\}$. The parameter $\nu$ was selected in grid
TABLE I

THE DETAILS OF EXPERIMENTAL DATASETS, \#NORMAL,\#ABNORMAL, AND D ARE NUMBER OF NORMAL, ABNORMAL DATA AND DIMENSIONALITY OF INPUT SPACE RESPECTIVELY

\begin{tabular}{|c|c|c|l|}
\hline Datasets & \#normal & \#abnormal & \#d \\
\hline Australian & 307 & 383 & 14 \\
\hline Breast Cancer & 444 & 239 & 10 \\
\hline Astroparticle & 2000 & 1089 & 4 \\
\hline Dna & 464 & 485 & 180 \\
\hline Sonar & 97 & 111 & 67 \\
\hline Ionosphere & 255 & 126 & 34 \\
\hline Bioinformatics & 221 & 117 & 20 \\
\hline Diabetes & 500 & 268 & 8 \\
\hline Liver & 200 & 145 & 6 \\
\hline SvmGuide3 & 296 & 947 & 22 \\
\hline Splice & 517 & 483 & 60 \\
\hline Vehicle & 212 & 217 & 18 \\
\hline Delf Pump & 1124 & 376 & 64 \\
\hline USPS & 1194 & 6097 & 256 \\
\hline
\end{tabular}

TABLE II

THE EXPERIMENTAL RESULT SHOWS THE CROSS VALIDATION ACCURACIES FOR FMSVDD, AND SVDD ON 14 DATASETS

\begin{tabular}{|c|c|c|l|}
\hline Datasets & SVDD & FMSVDD & \#Spheres \\
\hline Australian & $88 \%$ & $92 \%$ & 7 \\
\hline Breast Cancer & $90 \%$ & $98 \%$ & 7 \\
\hline Astroparticle & $94 \%$ & $90 \%$ & 7 \\
\hline Dna & $90 \%$ & $88 \%$ & 3 \\
\hline Sonar & $79 \%$ & $76 \%$ & 2 \\
\hline Ionosphere & $87 \%$ & $97 \%$ & 9 \\
\hline Bioinformatics & $85 \%$ & $99 \%$ & 3 \\
\hline Diabetes & $90 \%$ & $98 \%$ & 5 \\
\hline Liver & $88 \%$ & $92 \%$ & 2 \\
\hline SvmGuide3 & $87 \%$ & $96 \%$ & 9 \\
\hline Splice & $86 \%$ & $92 \%$ & 2 \\
\hline Vehicle & $89 \%$ & $88 \%$ & 2 \\
\hline Delf Pump & $89 \%$ & $96 \%$ & 7 \\
\hline USPS & $93 \%$ & $95 \%$ & 9 \\
\hline
\end{tabular}

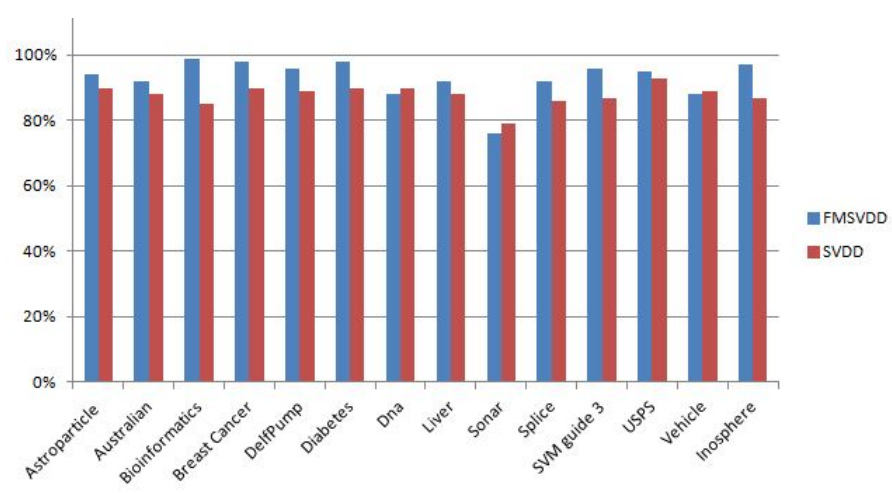

Fig. 1. Experimental results on 14 datasets

$\{0.1 i: i=1, \ldots, 9\}$. The number of hyperspheres $m$ was searched in grid $\{2,3,5,7,9\}$. The parameter $\alpha$ was set to 1.5 .

The results in table II and figure 1 show that FMSVDD outperforms SVDD on most of experimental datasets. Table II also presents the best number of hyperspheres for each dataset. 


\section{CONClusion}

We have presented a new fuzzy approach to Multi-sphere Support Vector Data Description to deal with datasets having distinctive data distributions. The fuzzy membership function of a data point represents the degree of belonging of that data point to a hypersphere. We have proved that structural risk could be reduced after each iteration in the learning process and the learning model would converge to a stable configuration. The experiment on 14 datasets in UCI repository has showed better performance of our proposed model in comparison to the standard SVDD.

\section{REFERENCES}

[1] D.Tax and R. P.Duin. "Support vector data description", Machine Learning, vol. 54, pp. 45-56, 2004.

[2] Y.Xiao, B.Liu, L.Cao, X.Wu, C.Zhang, Z.Hao, F.Yang, and J.Cao, "Multi-sphere Support Vector Data Description for Outliers Detection on Multi-Distribution Data", In Proc. IEEE International Conference on Data Mining Workshops, pp. 82-88, 2009.

[3] M.Yu and J.Ye, "A Small Sphere and Large Margin Approach for Novelty Detection Using Training Data with Outliers", IEEE Transaction on Pattern Analysis and Machine Intelligence, 31, 2088-2092, 2009.

[4] T. Le, D. Tran, W. Ma, and D. Sharma, "An Optimal Sphere and Two Large Margins Approach for Novelty Detection", In Proc. IEEE World Congress on Computational Intelligence (WCCI), pp. 909-914, 2010.

[5] P. Y.Hao and Y.H.Liu, "A New Multi-class Support Vector Machine with Multi-sphere in the Feature Space”, In Proc. IEA/AIE 2007, LNAI 4570, pp. 756-765, Springer, 2007.

[6] T. Le, D. Tran, P. Nguyen, W. Ma and D. Sharma, "Multiple Distribution Data Description Learning Method for Novelty Detection", In Proceedings of The International Joint Conference on Neural Networks (IJCNN), 2011, pp. 2321-2326.
[7] G. G.Towel, "Local expert autoassociator for anomaly detection" In: Proc. 17th International Conference on Machine Learning, pp. 10231030, Morgan Kaufmann Publishers Inc., 2000.

[8] V.Vapnik, The nature of statistical learning theory. Springer Verlag, 1995.

[9] J.Vert and J. P.Vert, Consistency and convergence rates of one class svm and related algorithm. Journal of Machine Learning Research, 7, 817-854, 2006.

[10] C. M.Bishop, Novelty detection and neural network validation. In: IEEE Proceedings of Vision, Image and Signal Processing, pp. 217-222, 1994

[11] V.Barnett and T.Lewis, Outliers in statistical data. 3rd ed: Wiley, 1978.

[12] C.Campbell and K. P.Bennet, A linear programming approach to novelty detection. Advances in Neural Information Processing Systems, vol. 14 2001.

[13] C. C.Chang and C. J.Lin, LIBSVM: A Library for Support Vector Machines.

[14] M.Kubat and S.Matwin, Addressing the curse of imbalanced training set: One-sided selection. In: Proc. 14th International Conference on Machine Learning, pp. 179-186, 1997.

[15] Y.Lin, Y.Lee, and G.Wahba, Support vector machine for classification in nonstandard situations. Machine Learning, vol. 15, pp. 1115-1148, 2002.

[16] M. M.Moya, M. W.Koch, and L. D.Hostetler, One-class classifier networks for target recognition applications. In: Proceedings of world congress on neural networks, pp. 797-801, 1991.

[17] T.Mu and A. K.Nandi, Multiclass Classification Based on Extended Support Vector Data Description. IEEE Transactions on Systems, Man, And CyberneticsPart B: Cybernetics, vol. 39, no. 5, pp. 1206-1217, 2009.

[18] L.Parra, G.Deco, and S.Miesbach, Statistical independence and novelty detection with information preserving nonlinear maps. Neural Computation, vol. 8, pp. 260-269, 1996.

[19] S.Roberts and L.Tarassenko, A Probabilistic Resource Allocation Network for Novelty Detection. Neural Computation, vol. 6, pp. 270-284, 1994.

[20] B.Schölkopf and A. J.Smola, Learning with kernels. Cambridge: The MIT Press, 2002. 